\title{
Use of Analytical Balances
}

\author{
Lane C. Sander \\ National Institute of Standards and Technology, \\ Gaithersburg, MD 20899, USA \\ lane.sander@nist.gov
}

Video DOI: http://doi.org/10.18434/T4DK5T

Key words: accuracy; balance; mass; quantitative analysis; weighing liquids; weighing solids.

Accepted: December 1, 2016

Published: January 12, 2017

https://doi.org/10.6028/jres.122.010

\section{Summary}

The determination of mass is a fundamental component in quantitative analysis, and weighing accuracy is an important factor for most applications. This presentation will highlight the use of different types of analytical balances and will demonstrate weighing operations for solids and liquids. Topics include calibration procedures, operation of mechanical and electronic balances, use of weighing vessels for solids, use of gas tight syringes for liquids, and best practices for solution preparation. Strategies are presented to minimize errors associated with evaporation or other sample losses that might occur with the determination of mass by difference. ${ }^{1}$

\footnotetext{
${ }^{1}$ Contribution of the National Institute of Standards and Technology. Not subject to copyright. Certain commercial equipment, instruments, or materials are identified to specify adequately the experimental procedure. Such identification does not imply recommendation or endorsement by the National Institute of Standards and Technology, nor does it imply that the materials or equipment identified are the best available for the purpose.
} 